\title{
The Soul - From a Religious-Philosophical Conjecture, Towards a Scientific-Medical Matter
}

\begin{abstract}
The present paper explored a thorny but needful field, the possibility of the existence of the soul and the resulting implications for medicine. Authors do not have the audacity to exhaust the subject and to bring an ultimate true. The text listed some baffling reports about consciousness phenomena that cannot be fully explained on a material basis. In order to fill the gap in knowledge, the authors venture an informed statement about the possible nature and characteristics of the soul. Lastly, the text cogitates the implications for medicine from the assumption of the existence of the soul. The authors excelled on the effort to avoid religious or philosophic vices that could pervert the reasoning. Disagreement from some readers is naturally expected, thus collaboration to improve this construct is more than welcome.
\end{abstract}

Keywords: Medicine and religion; Religion and science; Humanities; Medical philosophy; Spiritual therapies; Metaphysical mind body relation

Commentary
Volume 9 Issue 4 - 2017
Marcelo Saad ${ }^{1}$ *, Roberta de Medeiros ${ }^{2}$ and
Amandla Cristina Mosini ${ }^{3}$
${ }^{1}$ President, Spiritist Medical Association of S. Paulo, Brazil
${ }^{2}$ Professor of Physiology, Centro Universitario S. Camilo, Brazil
${ }^{3}$ Master's degree student, Universidade Federal de S. Paulo,
Brazil
*Corresponding author: Marcelo Saad, Spiritist Medical
Association of S. Paulo, Rua Pedro Severino Jr., 323. ZIP
04310-060. S. Paulo, SP Brazil, Tel/Fax: +55 11 2574-8696;
Email: msaad@uol.com.br
Received: September 18, 2017 | Published: November 24,
2017

\section{Anomalous Phenomena Challenging the Existent Models}

From a conventional scientific perspective, the existence of the soul might be considered improbable or paradoxical. However, many recent studies with correct methodology have documented disconcerting events and experiences related to anomalous phenomena of consciousness. Unexplained events related to mystical experiences are regularly reported in the medical literature $[4,5]$. On biomedical grounds, there is an increasing number of researches to explore the idea that mind is a separate entity and can existing outside the brain [6]. This assumption has been called the possibility of consciousness independent of the body. The phenomena that rise medical concerns will belong to one of the three groups below:

\section{Spiritual phenomena during specific altered states of consciousness}

One of them, the near-death experience, is the set of reports from many survivors of cardiac arrest during the period of unconsciousness. Some of the sensory perceptions could be attributed to hallucinations by uncontrolled release of neurotransmitters under this extreme situation [7]. However, it does not explain how a few individuals can precisely describe events occurred during resuscitation [8], since the brain stops processing electrical impulses without oxygen. This is a potential evidence of an out-of-body experience. Other examples of spiritual phenomena during altered states of consciousness are end-of-life experiences, which include visions of dead relatives, odd coincidences and behavioral changes towards family rifts. These phenomena are not uncommon, and they differ from druginduced hallucinations, occurring in clear consciousness [9]. possibility of the existence of the soul, along with the implications for a whole vision of human being. 


\section{Anomalous reception of information}

Mediumship can be defined as an experience in which an individual (the medium) intends to be in communication with the personality of a deceased person (a spirit) [10]. The precision of some information obtained cannot be attributed to chance, since it is accurate and specific, without prior knowledge, in the absence of sensory feedback, and without using deceptive means [11]. For example, a study [12] of 13 texts transmitted by the spirit of a deceased person found dozens of verifiable items, and $98 \%$ of them had clear and precise match. A research with functional magnetic resonance [13] evidenced that mediumistic psychography is different from writing a mere essay. Ten experienced mediums were evaluated in two different conditions: during trance of psychography and during writing freely. Comparing the two situations, there were significant differences in the activities of brain areas and in the complexity of the produced texts.

\section{Memories of alleged past lives}

Suggestive of reincarnation. A few researchers dedicated efforts to study cases of subjects who have claimed to remember previous lives. Some of such statements have been verified after investigations of data that the subject would have not accessed. Once again, the subject with the mark has no verifiable connection to the other person. Many published cases cannot be explained by chance or fraud, since the information provided by subjects is precise and spontaneous [14]. Among more than 2,500 documented and studied cases of children with supposed past lives memories, one or more characteristics are present in many cases [15]: the revelations corresponded to a particular deceased person; presence of birth marks corresponding to wounds on the body of the previous personality; behaviors related to their former life (phobias, philias, and attachments); recognition of people and places from the previous life, even under controlled conditions.

\section{A Tentative Construct for The Soul Based Upon Scientific Method}

Some phenomena that seem to counteract the common sense of reality are genuine calls for the discussion about the soul. Phenomenological descriptions of mystical experiences, as well as other unusual (or exceptional) mind-matter anomalies suggest that this aspect of the problem needs reconsideration [16]. The very existence of spiritual phenomena, summed up with its similarities among diverse cultures and epochs, calls up to a more enlightened scientificism. The true science encompasses deep and respectful understanding of the work of others, humility before empirically demonstrable abnormalities, and openness to new ideas.

Some scholars anticipate the need for a transition to a new scientific paradigm, since some data suggest the survival of consciousness after death, and the existence of non-physical levels of reality [17]. Academic researchers linked to this ideal regularly publish their research on reputable peer-reviewed medical journals, although on an individual basis. Notwithstanding, there is a need to sew all this knowledge in a continuous fabric, in a way the product would be acknowledged by science. In scientific method, there is a natural progression from conjecture to hypothesis and to theory.

A conjecture is a proposition based on incomplete information. It is an incomplete model, or an analogy to another domain. A conjecture may surge from the observation of something curious or odd from nature. Existing a conjecture, the next step in scientific method is to pose the hypothesis, a proposition that can be tested by observations or experiments. The hypothesis is a rational speculation, based upon an observed event or phenomenon, It is a tentative prediction about the connection between one thing and one other thing (such as "if one do ' $X$ ', then ' $Y$ ' will happen). Next comes the experiment, where the predictions will be confronted to the obtained empirical data, in order to accept or not the assumption. As the hypothesis is a reasoning assumption, it can be wrong, thus it can be disproven.

Following the above steps for the case of the soul, there is already a conjecture, since many ancient practices, religious traditions and contemplative approaches have their conceptions to interpret its existence and its characteristics. For them, the conjecture of existence of the soul were a need to explain observed natural phenomena. Now, to evolve to a hypothesis, it is necessary to design an experiment to test it. However, the phenomena attributed to the soul have scarce and irregular manifestations. Maybe it happens because we currently have no means to evocate properties of this subtle material in a controlled and predictable way. All scientific reports about spiritual phenomena are related to spontaneous eruptions of this kind that once in a while comes to our knowledge.

Even without data from prospective experiments, there are still the anecdotic observations of spiritual phenomena. This literature may enable the advance towards a model of the soul based upon the scientific method. The next step is to formulate a theory, the attempt to explain what has been observed. What is important in a theory is to be internally consistent, based upon available evidence, and effective in problem-solving. The amount of supporting data is not the most important factor, since a theory is the best model for a given epoch. Thus, the present text launches below a statement about the possible nature and characteristics of the soul. Although it may seem to have religious or philosophic ideas, it is constructed upon the facts already discussed on this paper:

"The soul, also taken as spirit, may be understood as the transpersonal, ethereal and immaterial essence of the individual. The soul must be responsible for all mind properties, which are manifested on our material reality by brain activity. The soul has a broader consciousness that the one manifested by the person's mind, and in some states it can capture information without the physical senses. The soul may continue to exist after the death of brain, although in a condition we cannot easily understand and access. In such state, the soul may keep its individuality and everything that characterized the person on the previous experience. This free soul seems capable to make contact with some people's minds and, under specific conditions, to transmit consistent information. The nature of the soul may be related to an immaterial reality that is still beyond our capacity of access. 


\section{Medical Impacts of the Existence of the Soul}

The discussion on the existence of the soul, although challenging and provocative, may produce high scientific implications, many of them with medical consequences. Currently, the basic assumptions of what can influence health and disease do not consider the participation of the soul. A paradigm that include this dimension would broaden the classification of diagnostics and treatments, particularly concerning complementary therapies [18]. A true bio-psycho-social-spiritual interpretation of the human being would emerge, with profound transformations on concepts of health, disease, treatment and cure.

A natural utility of mediumship is to comfort people who lost beloved ones through communication with the soul of the deceased person. People would embrace this approach with more confidence if science could develop methods to check authenticity of the writings. If science accomplishes such achievement, mediumship would have yet another utility. An intriguing essay [19] studied a psychographic writing from the 1940's about some biological functions that were known then. The text describes many functions of the pineal gland, at the time taken as an unimportant structure. Decades later, all the melatonin functions were discovered, most of which coinciding with the mediumistic text. What if, in future, we could rely on novel technical information brought by mediumship, aiming advancement of knowledge?

In other direction, the concept of continuity of the soul after physical death may improve mental health approaches. If immortality were scientifically plausible, the fear of death of a large number of people would be lessen and made more tolerable. Further benefit for mental healthcare would come from the idea of reincarnation. The hypothesis of previous lives can contribute to further understanding of some disorders in medicine and psychology not fully explicable by genetics and environmental influences. Some examples include severe phobias and philias in early infancy, and unusual behavior manifested soon after birth [20]. For such cases, the Past-Life Regressive Therapy aims on restructuring of a trauma that can rest in a supposed past life, through a hypnotic-like altered consciousness state. Problematic personal relationships, phobias, and a lack of meaning and purpose in life have been the conditions most successfully treated with this therapy [21].

Understanding spiritual experiences will broaden our understanding of human nature, enhance our cultural sensitivity, and improve the effectiveness of mental health initiatives [22]. There is a need to take into account the cultural environment and belief systems of psychotherapy patients. Psychotherapeutic approaches should take account of the belief in life after death held by most of the world's population. Respect for patient opinions and subjective realities is a therapeutic need and an ethical duty, even though therapists may not share the same beliefs [23].

\section{Conclusion}

The present paper explored a thorny but needful field, the possibility of the existence of the soul and the resulting implications for medicine. Authors do not have the audacity to exhaust the subject and to bring an ultimate true. The text listed some baffling reports about consciousness phenomena that cannot be fully explained on a material basis. In order to fill the gap in knowledge, the authors venture an informed statement about the possible nature and characteristics of the soul. Lastly, the text cogitates the implications for medicine from the assumption of the existence of the soul. The authors excelled on the effort to avoid religious or philosophic vices that could pervert the reasoning. Disagreement from some readers is naturally expected, thus collaboration to improve this construct is more than welcome.

\section{Conflict of Interest}

Authors declare there are not any actual or potential conflicts of interest.

\section{Acknowledgement}

Authors are grateful to the Spiritist Medical Association of S. Paulo (Brazil) for the encouragement and support to the accomplishment of this manuscript.

\section{References}

1. Dolan B (2007) Soul searching: a brief history of the mind/body debate in the neurosciences. Neurosurg Focus 23(1): E2.

2. Perakis CR (2013) What about the Soul? Academic Medicine 88(10): 1521.

3. Santoro G, Wood MD, Merlo L, Anastasi GP, Tomasello F, et al. (2009) The anatomic location of the soul from the heart, through the brain, to the whole body, and beyond: a journey through Western history, science, and philosophy. Neurosurgery 65(4): 633-643.

4. Bobrow RS (2003) Paranormal phenomena in the medical literature sufficient smoke to warrant a search for fire. Med Hypotheses 60(6): 864-868.

5. Bonilla E (2010) Mind-body connection, parapsychological phenomena and spiritual healing - a review. Invest Clin 51(2): 209238.

6. Daher JC Jr, Damiano RF, Lucchetti AL, Moreira-Almeida A, Lucchetti G (2017) Research on Experiences Related to the Possibility of Consciousness Beyond the Brain: A Bibliometric Analysis of Global Scientific Output. J Nerv Ment Dis 205(1): 37-47.

7. Agrillo C (2011) Near-Death Experience: Out-of-Body and Out-ofBrain? Review of General Psychology 15(1): 1-10.

8. Parnia S, Spearpoint K, de Vos G, Fenwick P, Goldberg D, et al. (2014) AWARE-AWAreness during REsuscitation-a prospective study. Resuscitation 85(12): 1799-17805.

9. Fenwick P, Lovelace H, Brayne S (2010) Comfort for the dying: five year retrospective and one year prospective studies of end of life experiences. Archives of Gerontology and Geriatrics 51(2): 173-179.

10. Moreira-Almeida A, Santos FS (2012) Exploring frontiers of the mind-brain relationship. Springer Publisher, New York, USA.

11. Beischel J, Boccuzzi M, Biuso M, Rock AJ (2015) Anomalous information reception by research mediums under blinded conditions II: replication and extension. Explore (NY) 11(2): 136142.

12. Rocha AC, Paraná D, Freire ES, Lotufo Neto F, Moreira-Almeida A (2014) Investigating the fit and accuracy of alleged mediumistic writing: a case study of Chico Xavier's letters. Explore (NY) 10(5): 300-308. 
13. Peres JF, Moreira-Almeida A, Caixeta L, Leao F, Newberg A (2012) Neuroimaging during trance state: a contribution to the study of dissociation. PLoS One 7(11): e49360.

14. Tucker JB (2008) Children's reports of past-life memories: a review. Explore (NY) 4(4): 244-248.

15. Bonilla E (2015) Evidence that suggest the reality of reincarnation Invest Clin 56(2): 215-240.

16. Walach H (2007) Mind - Body - Spirituality. Mind \& Matter 5(2): 215-240

17. Beauregard M, Schwartz GE, Miller L, Dossey L, Moreira-Almeida A et al. (2014) Manifesto for a post-materialist science. Explore (NY) $10(5): 272-274$

18. Tataryn DJ (2002) Paradigms of health and disease: a framework for classifying and understanding complementary and alternative medicine. J Altern Complement Med 8(6): 877-892.
19. Lucchetti G, Daher JC Jr, Iandoli D Jr, Gonçalves JP, Lucchetti AL (2013) Historical and cultural aspects of the pineal gland: comparison between the theories provided by Spiritism in the 1940s and the current scientific evidence. Neuro Endocrinol Lett 34(8): 745-755.

20. Stevenson I (2000) The phenomenon of claimed memories of previous lives: possible interpretations and importance. Med Hypotheses 54(4): 652-659.

21. Simoes M (2002) Altered States of Consciousness and Psychotherapy - A Cross-Cultural Perspective. Int J Soc Psychiatry 21(1): 145-152.

22. Moreira-Almeida A (2009) Differentiating spiritual from psychotic experiences. BJ Psych 195(4): 370-371

23. Peres JF (2012) Should psychotherapy consider reincarnation? J Nerv Ment Dis 200(2): 174-179. 